\title{
Infinite Time Irreversible Processes
}

\author{
José C. Íñiguez ${ }^{1}$ \\ ${ }^{1} 1227$ 21st Street, Douglas AZ 85607, USA \\ Correspondence: José C. Íñiguez, 1227 21st Street, Douglas AZ 85607, USA. Tel: 1-602-368-1111. E-mail: \\ iniguez.jose@gmail.com
}

Received: November 13, 2012 Accepted: December 13, 2012 Online Published: January 15, 2013

doi:10.5539/ijc.v5n1p20

URL: http://dx.doi.org/10.5539/ijc.v5n1p20

\begin{abstract}
Through a thermodynamic argument based on Planck's as well as the total entropy irreversibility criterions it is possible to prove that contrary to common wisdom, infinite-time irreversible processes constituted by a succession of equilibrium states in which all the thermodynamic properties are defined and quantifiable, are possible. As such, these irreversible processes are capable of graphical representation in thermodynamic state space. It is also shown that these infinite-time irreversible processes are thermodynamically equivalent to their finite-time counterparts. Not only do these results demand a revision of our current conceptualization of irreversibility; they also bring a purely thermodynamic alternative to the postulate of local equilibrium for the thermodynamic analysis of the evolutionary path of irreversible processes.
\end{abstract}

Keywords: thermodynamics, entropy, lost work, reversibility, irreversibility, time, state space, local equilibrium

\section{Introduction}

The most general criterion of irreversibility offered by thermodynamics takes form in the total entropy increase principle: “... Any actual or irreversible process is characterized by an increase in the total entropy of all systems concerned." (Lewis \& Randall, 1961). This statement finds re-expression in a number of equivalent propositions (Schmidt, 1966), one of which takes form in Planck's principle of the impossibility of recuperation of the original condition of the universe: “... A process which can in no way be completely reversed is termed irreversible, all other processes reversible. That a process may be irreversible, it is not sufficient that it cannot be directly reversed... The full requirement is, that it be impossible, even with the assistance of all agents in nature, to restore everywhere the exact initial state when the process has once taken place" (Planck, 1990).

Notwithstanding the generality of the previous statements, a number of subsidiary characteristics are commonly ascribed to these processes. Thus, irreversible processes are commonly described as finite-time processes (Graetzel \& Infelta 2002) comprised by a temporal succession of non-equilibrium states in which some of the relevant thermodynamic state functions such as $T, P$, and $S$ are neither defined nor quantifiable (Tolman \& Fine, 1948; Kestin, 1991; Vavruch, 2002; Garfinkle, 2002), situation that hinders their representation as continuous graphs in state space (Schmidt, 1966).

The argument to be introduced below based on Planck's as well as the entropy increasing criterions of irreversibility shows, however, that these subsidiary conditions are not general; that infinite time irreversible processes constituted by a succession of equilibrium states and capable of graphical representation are indeed possible; that an infinite-time isothermal irreversible process is thermodynamically equivalent to its irreversible finite-time counterpart; and that this equivalence constitutes an alternative approach to that embodied by the postulate of local equilibrium for the thermodynamic analysis of irreversible processes.

These results will be obtained by way of proving that an infinite-time irreversible process can be generated via the concatenation of its reversible counterpart-the reversible version of the original irreversible process-in which an amount of work $W_{r e v}$ is produced, with a work degrading step in which $W_{r e v}$ is degraded as heat at the temperature of a heat reservoir. The fact that in the said concatenation the irreversible process comes about at the conclusion of its reversible counterpart makes it an infinite-time irreversible process. The proof that this infinite-time irreversible process is thermodynamically equivalent to its irreversible finite-time counterpart rests on the fact that the total entropy change for the two paths mentioned is one and the same. 


\section{Entropy Production as a Gauge of Lost Work in Irreversible Processes}

According to Planck (1990),

“... The second law of thermodynamics states that there exists in nature for each system of bodies a quantity, which by all changes of the system either remains constant (in reversible processes) or increases in value (in irreversible processes). This quantity is called, following Clausius, the entropy ..."

According to Smith and Van Ness (1965),

“... Actual processes are irreversible, and every irreversibility results in lost work ..."

The fact that according to these statements every irreversible process is accompanied by the production of entropy as well as the production of lost work, strongly suggests the existence of a connection between these two magnitudes. That this is indeed the case is the matter of the argument that follows which compares the effects of the reversible (rev) evolution of a thermodynamic system $(s)$ from a given initial to a given final condition, with those produced when the evolution between the same two states takes place irreversibly (irr). In these processes the surroundings take the form of a heat bath of temperature $T$, and a mechanical reservoir. Combined, system and surroundings, define the universe $(u)$ of these processes.

Let us start by writing the following equations for the internal energy change $(\Delta U)$ of the system along each of these evolving paths

$$
\begin{gathered}
\Delta U_{s, r e v}=Q_{s, r e v}-W_{r e v} \\
\Delta U_{s, i r r}=Q_{s, i r r}-W_{i r r}
\end{gathered}
$$

In the previous equations $W_{r e v}$ and $W_{i r r}$ represent, respectively, the work outputs along the reversible and irreversible paths. The fact that heat lost by the system is heat absorbed by the bath, and vice versa

$$
Q_{s}=-Q_{\text {bath }}
$$

allows us to write Equations (1) and (2) as follows

$$
\begin{gathered}
\Delta U_{s, r e v}=-Q_{b a t h, r e v}-W_{r e v} \\
\Delta U_{s, i r r}=-Q_{b a t h, i r r}-W_{i r r}
\end{gathered}
$$

In Equations (4) and (5) the terms $Q_{\text {bath,rev }}$ and $Q_{\text {bath,irr }}$ refer, respectively, to the heat exchanged between the bath and the system when the latter evolves along the reversible path, and that exchanged when the system's evolution follows the irreversible path. As known (Denbigh 1968), the entropy change for the heat bath is determined by the quotient of the actual amount of heat by it exchanged divided by its temperature, with independence of the nature of the process in which such a heat originated, or to which such a heat is destined.

The fact that the initial and final conditions of the system are the same for the reversible and irreversible evolutions being considered, combined with the fact that the internal energy is a state function, leads to

$$
\Delta U_{s, \text { rev }}=\Delta U_{s, i r r}
$$

Introduction of this condition in Equations (4) and (5) produces, after rearrangement, the following expression

$$
W_{\text {lost }}=W_{\text {rev }}-W_{\text {irr }}=Q_{\text {bath,irr }}-Q_{\text {bath }, \text { rev }}
$$

Equation (7) makes it clear that the lost work $\left(W_{\text {lost }}\right)$, defined as the difference between the reversible and irreversible work outputs

$$
W_{\text {lost }}=W_{\text {rev }}-W_{\text {irr }}
$$

appears as that extra amount of heat rejected to the heat bath in excess of that rejected in the reversible path.

Upon division by $T$, Equation (7) transforms into

$$
W_{\text {lost }} / T=\left(Q_{\text {bath,irr }} / T\right)-\left(Q_{\text {bath,rev }} / T\right)=\Delta S_{\text {bath,irr }}-\Delta S_{\text {bath }, \text { rev }}
$$

The following equations will now be written for the entropy changes of the universes of these two paths

$$
\begin{aligned}
& \Delta S_{u, r e v}=\Delta S_{s, r e v}+\Delta S_{b a t h, r e v} \\
& \Delta S_{u, i r r}=\Delta S_{s, i r r}+\Delta S_{b a t h, i r r}
\end{aligned}
$$

The same reasoning leading to Equation (6) is here used to write

$$
\Delta S_{s, r e v}=\Delta S_{s, i r r}
$$


Substitution of Equation (12) in (11) leads to

$$
\Delta S_{u, i r r}=\Delta S_{s, r e v}+\Delta S_{\text {bath }, i r r}
$$

Once Equation (10) is solved for $\Delta S_{s, r e v}$, and the resulting expression substituted in Equation (13), we get

$$
\Delta S_{u, i r r}=\Delta S_{u, r e v}+\Delta S_{\text {bath, irr }}-\Delta S_{\text {bath }, \text { rev }}
$$

The adequate combination of Equations (9) and (14) leads to

$$
\Delta S_{u, i r r}=\Delta S_{u, r e v}+W_{l o s t} / T
$$

And the fact that $\Delta S_{u, r e v}=0$, to

$$
\Delta S_{u, i r r}=W_{\text {lost }} / T
$$

Equation (15) states that the total or universe entropy change for an isothermal irreversible process is equivalent to the summation of the entropy changes of its reversible counterpart, in which an amount of work $W_{\text {rev }}$ is produced, plus that of the process in which this work is degraded as heat at the temperature of the heat reservoir. Equation (16), obtained combining Equation (15) with the known fact that the total entropy change for a reversible process is zero, identifies the entropy change as a gauge or measure of the work lost in irreversible processes. Let us also point out that constancy of temperature was the only restriction introduced in the thermodynamic argument leading to Equations (15) and (16). Consequently, no other limitation than this applies to the use of these equations. Alternative derivations leading to the result shown in Equation (16) can be found in the literature (Smith \& Van Ness, 1965; Balzhiser et al., 1974; Di Liberto, 2007).

\section{The Degradation of Work and the Transition from Reversibility to Irreversibility}

"The significance of the second law of thermodynamics depends on the fact that it supplies a necessary and far reaching criterion as to whether a definite process which occurs in nature is reversible or irreversible ... A process which can in no way be completely reversed is termed irreversible, all other processes reversible. That a process may be irreversible, it is not sufficient that it cannot be directly reversed ... The full requirement is, that it be impossible, even with the assistance of all agents in nature, to restore everywhere the exact initial state when the process has once taken place" (Planck, 1990).

To understand the significance of Equation (15) let us consider the reversible and isothermal expansion of an ideal gas from an initial condition $\left(P_{i}, V_{i}\right)$ to a final condition $\left(P_{f}, V_{f}\right)$ as represented in Figure 1(b). The effect of this process, the first step of the concatenation made explicit by the right hand side of Equation (15), takes form in the transformation of an amount of heat $Q_{\text {rev }}$ absorbed from the heat bath of temperature $T$, into an equivalent amount of work $W_{\text {rev }}=n R T \ln \left(V_{f} / V_{i}\right)$ deposited in the mechanical reservoir. Let us now point out here two facts regarding the expansion that has just taken place: First, that on reason of its reversibility, the entropy change for the universe of this process is equal to zero; Second, that also on reason of its reversibility, the transit of the ideal gas from its initial to its final condition can be represented as a continuous path in state space; here in the form of a trajectory on a $P-V$ graph. Let us now retrieve from the mechanical reservoir the amount of work there previously deposited, and degrade it as an equivalent amount of heat at the temperature of the heat bath. This action embodies, it should be clear, the second step of the concatenation previously noted. Let us now point out four facts in regard to the just performed work-to-heat degradation. First, that in accord with Equation (16), this work degrading process is accompanied by a total entropy increase of magnitude $W_{\text {lost }} / T=W_{\text {rev }} / T=n R \ln \left(V_{f} / V_{i}\right)$. Second, that in confirmation to that expressed by Equation (15), the addition of this entropy change-corresponding to process 1 (c)- to that of magnitude zero corresponding to its reversible antecedent-process 1(b)- produces a total entropy change of magnitude $n R \ln \left(V_{f} / V_{i}\right)$, identical to that known to correspond to the isothermal irreversible counterpart represented in Figure 1(a) consisting in an ideal gas free-expansion transiting between the same initial and final states (Klotz \& Rosenberg, 1986). Third, the fact that in only involving the heat and mechanical reservoirs, the degradation of $W_{\text {rev }}$ has no effect on the values of the thermodynamic variables $(P, V)$ defining the state of the gas. At the end of the degradation of $W_{\text {rev }}$ the gas remains at its final condition $\left(P_{f}, V_{f}\right)$. Fourth, the realization that the moment the work degrading process takes place, the universe of the reversible expansion becomes irreversible. The reason is simple and subsumed in Planck's quote given at the beginning of this section. As long as $W_{r e v}$ is available we can use it to reverse each and every change produced in the reversible expansion, and in doing so bring the right hand side universe back to its precise original condition, without changes in other bodies remaining. Once $W_{\text {rev }}$ is lost, however, the compression of the gas back to its initial condition can only be performed at the expense of 
leaving a permanent change in at least that body called to supply the missing work. Our inability to recover the original condition of the universe without changes in other bodies remaining is what makes the universe of this concatenation irreversible. The reversibility of the expansion, guaranteed by the availability of $W_{\text {rev }}$, turns into irreversibility the moment this work is degraded as heat, and when this happens, the universes at both ends of the equal sign in Figure 1 become identical.

Keeping the previous description in mind it shouldn't be difficult to understand that if starting from its initial condition the expansion is carried on as a succession of reversible expansion steps of infinitesimal extent, degrading at the conclusion of each of them the reversible work there produced, a reversible to irreversible transition will be experienced by each and every point of the expansion's path without otherwise affecting the values of the state functions already in place (entropy excluded), and in being this so, at the conclusion of this process the continuous $P-V$ curve originally describing the evolving path of a reversible process will become the path of an irreversible process. The fact that each and every point of the irreversible path thus defined can only come about after its reversible antecedent has taken place, an event demanding an infinite time, leads to the inescapable conclusion that the said irreversible path will also demand an infinite time. Furthermore, the identical entropy changes produced by the processes at both sides of the equal sign in Figure 1 leads to the realization that the finite-time irreversible process represented in 1(a) is thermodynamically equivalent to the infinite time irreversible process produced by the concatenation of Figure 1(b) with Figure 1(c).

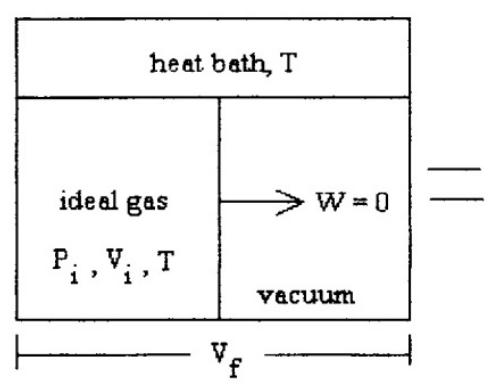

(a)

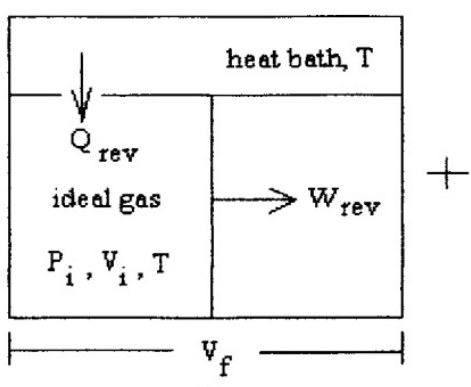

(b)

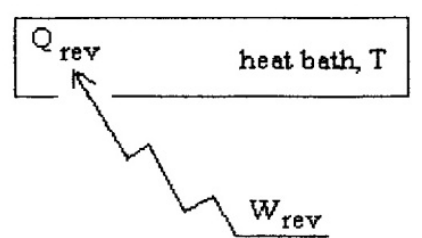

(c)

Figure 1. The universe of the irreversible and isothermal ideal gas free-expansion shown in (a) is equivalent to that defined by the concatenation of its reversible counterpart, the reversible and isothermal ideal gas expansion shown in (b), with the work degrading process shown in (c)

It should be obvious that a similar line of reasoning can be applied to any other isothermal irreversible process, and that different types of graphs can be targeted besides the one chosen above to exemplify the concept. Some of these graphs, like an $S_{u}-v s-\xi$, -with $\xi$ representing the degree of advancement of the process being considered- will be continuous but certainly not coincident with that corresponding to the reversible universe. In this case each and every point in the continuous irreversible path will be displaced from its reversible counterpart in an amount $W_{\text {lost }, \xi} / T$, with $W_{\text {lost }, \xi}$ representing the total amount work degraded up to that point $\xi$ in the advancement of the process.

Another fact worth noticing in regard to Figure 1 is that in the universe of the free expansion there is no degradation of tangible work into heat, as is the case in the second step of the concatenation. The fact that the same entropy change applies for these two processes is a clear indication that the wasted dissipation of the work-producing potential taking place in the irreversible expansion, is equivalent or entropically indistinguishable from the actual degradation of tangible work into heat.

\section{Discussion}

"The admonishment of Brostow that thermodynamicists are mistaken in assuming that the scope and contents of thermodynamics are time independent has not been appreciated, neither by thermodynamicists, let alone by kineticists" (Garfinkle, 2000).

"Time has no role to play in settling issues of thermodynamics" (Radhakrishnamurty, 2010).

The fact that the analytical tools of classical thermodynamics are "... correct for equilibrium systems, for reversible (equilibrium) processes, and for processes between equilibrium states ..." (Vavruch, 2002), combined 
with the fact that an irreversible process is conceived as a temporal succession of non-equilibrium states in which most of the thermodynamic variables are nor defined nor quantifiable, leads to the realization that if a thermodynamic description of the path of irreversible processes is to be obtained it will have to be through the application of the said tools properly complemented with a number of assumptions (postulates). The classical approach to the thermodynamic description of irreversible processes is based on the Postulate of Local Equilibrium. In Garfinkle's version of this postulate for closed systems, an irreversible process consists of a succession of cells "... of short duration, with each occupying the whole system ..." (Garfinkle, 2002); The essential idea being, in the words of Moore, that " ... at a time t, the ... cell is isolated from its surroundings and allowed to come to equilibrium, so that at $t+\delta t$, the $\mathrm{P}$ and $\mathrm{T}$ of the cell can be specified. The Postulate of Local Equilibrium is that we can take the $\mathrm{P}$ and $\mathrm{T} \ldots$ in the original nonequilibrium system at time $\mathrm{t}$ to be equal to the $\mathrm{P}$ and $\mathrm{T} \ldots$ when equilibrium is reached at $t+\delta t$ " (Moore, 1972); here $\delta t$ represents what is called the time of relaxation, an extra-thermodynamic, kinetic concept. From the modus operandi of this postulate it is understood that the equilibrium values adopted by the relevant variables at $t+\delta t$ necessarily correspond to those of a point in the path of the reversible counterpart to the original irreversible process. By this postulate a finite-time irreversible process becomes, according to Kestin "... a reversible process which occurs at a finite rate ..." (Kestin, 1991). In reference to Figure 1 this postulate allows the use the continuous $P-V$ graph associated to the reversible expansion, to represent also that of the irreversible one.

The argument presented in section 3 has allowed us to realize that the thermodynamic description of the evolving path of isothermal irreversible processes via the continuous reversible graph finds sustenance in a purely thermodynamic argument devoid of non-thermodynamic notions such as the relaxation time or the relaxation rate. This argument managed to prove-via the reversible to irreversible switch produced in an isothermal process by a work degrading step-that the reversible locus is common to both of these processes, and if so that a sequence of equilibrium states in which all the pertinent thermodynamic variables are defined and quantifiable applies equally to reversible and irreversible processes.

In regard to the question of the role of time in thermodynamics, it serves to highlight the fact that thermodynamics finds irreversible process 1 (a) to be equivalent to the irreversible process coming out of the concatenation of 1(b) with 1(c) with independence of the fact that the former is a finite-time process while the latter is an infinite-time process. The time demanded by their respective occurrences-as different as two amounts of time can be-is inconsequential, i.e. plays no role in thermodynamics deciding their equivalence; a fact that constitutes evidence for positions such as Radhakrishnamurty's denying time any role in thermodynamics' affairs. In the same direction points the fact that the purely thermodynamic approach previously presented to treat isothermal irreversible processes as reversible makes unnecessary the time-related, extra-thermodynamic concepts of time and rate of relaxation.

The previous considerations suggest that in classical thermodynamics neither time nor the nature of the succession of states connecting the initial and final conditions of a given isothermal process can be considered as indicators of the reversible or irreversible nature of such process. If these subsidiary conditions were the only ones at our disposal for the assessment of irreversibility we would be forced to agree with Kestin's proposition “... that the distinction between reversible and irreversible processes ... be dispensed with entirely: the former being a special case of the latter, only lacking direction" (Garfinkle, 2000).

The fact that the introduction of the entropy function into the analysis of the expansions of Figure 1 brings forward two separate and distinct $S_{u}-v s-\xi$ graphs for the reversible and irreversible versions of this process unambiguously indicates that it is entropy, total entropy that is, the true thermodynamic criterion of irreversibility and not the subsidiary conditions previously referred.

\section{Conclusions}

Two main conclusions can be obtained from the previous discussions. First, that neither time nor the thermodynamic nature of the states along its path has any role in defining the irreversibility of a thermodynamic process. This task is the sole province of the total entropy increase principle. Second, the fact that the evolving path of any isothermal irreversible process can be associated with a succession of thermodynamically defined intermediate states constitutes a purely thermodynamic alternative to the postulate of local equilibrium for the analysis of such processes. 


\section{References}

Balzhiser, R. E., Samuels, M. R., \& Eliassen, J. D. (1974). Termodinámica Química para Ingenieros. México D.F.: Prentice Hall/Internacional.

Denbigh, K. (1968). The Principles of Chemical Equilibrium. Cambridge, UK: Cambridge University Press.

Di Liberto, F. (2007). Entropy Production and Lost Work for Irreversible Processes. Phil. Mag., 87, 569. http://dx.doi.org/10.1080/14786430600909006

Garfinkle, M. (2000). The Thermodynamic Natural Path in Chemical Reaction Kinetics. Discrete Dynamics in Nature and Society, 4, 145-164. http://dx.doi.org/10.1155/S1026022600000145

Garfinkle, M. (2002). A thermodynamic-Probabilistic Analysis of Diverse Homogeneous Stoichiometric Chemical Reactions. J. Phys. Chem., A106, 490-497. http://dx.doi.org/10.1021/jp013048n

Graetzel, M., \& Infelta, P. (2002). The Bases of Chemical Thermodynamics, Volume 1. Boca Raton, FL: Universal Publishers.

Kestin, J. (1991). Local-Equilibrium Formalism Applied to Mechanics of Solids, International Symposium on Micromechanics. Retrieved from http://www.osti.gov/bridge/servlets/purl/7206171-mJAsbG/7206171.pdf

Klotz, I. M., \& Rosenberg, R. M. (1986). Chemical Thermodynamics. Menlo Park, California: Benjamin/Cummings.

Lewis, G. N., \& Randall, M. (1961). Thermodynamics (2nd ed., Revised by K. S. Pitzer, \& L. Brewer). New York, NY: McGraw-Hill Book Company.

Moore, W. J. (1972). Physical Chemistry (4th ed.). Englewood Cliffs, NJ: Prentice-Hall.

Planck, M. (1990). Treatise on Thermodynamics. New York, NY: Dover.

Radhakrishnamurty, P. (2010). Maxwell's Demon and the Second Law of Thermodynamics. Resonance, 548-560. http://dx.doi.org/10.1007/s12045-010-0061-1

Schmidt, E. (1966). Thermodynamics: Principles and Applications to Engineering (3rd ed.). New York, NY: Dover.

Smith, J. M., \& Van Ness, H. C. (1965). Introduction to Chemical Engineering Thermodynamics. New York, NY: McGraw-Hill.

Tolman, R. C., \& Fine, P. C. (1948). On the Irreversible Production of Entropy. Reviews of Modern Physics, 20(1), 51-77. http://dx.doi.org/10.1103/RevModPhys.20.51

Vavruch, I. (2002). Conceptual Problems of Modern Irreversible Thermodynamics. Chem. Listy, 96, 271-275. Retrieved from http://www.chemicke-listy.cz/docs/full/2002_05_01.pdf 